\title{
Outcome of Early Neurological Rehabilitation Patients Colonized with Extended-Spectrum Beta-Lactamase (ESBL) Producing Bacteria
}

\author{
Jens D. Rollnik \\ Institute for Neurorehabilitation Research (InFo), Hannover Medical School (MHH), Hessisch Oldendorf, \\ Germany \\ Email: prof.rollnik@bdh-klinik-hessisch-oldendorf.de
}

Received 1 January 2015; accepted 13 January 2015; published 15 January 2015

Copyright (C) 2015 by author and Scientific Research Publishing Inc. This work is licensed under the Creative Commons Attribution International License (CC BY). http://creativecommons.org/licenses/by/4.0/

(c) (i) 0pen Access

\begin{abstract}
Colonization with multidrug-resistant germs, in particular methicillin-resistant Staphylococcus aureus (MRSA) and extended-spectrum beta-lactamase producing bacteria (ESBL), is an emerging threat in early neurological rehabilitation. This study examined whether colonization with ESBL bacteria had deteriorating effects on neurological rehabilitation patients because of contact precautions (CP). Medical records have been carefully reviewed with respect to colonization with ESBL, outcome variables (functional independence), morbidity, and length of stay (LOS). 148/643 $(23.0 \%)$ patients were ESBL positive on admission. ESBL carriers had a significantly longer LOS in early neurological rehabilitation $(67.5(42.0)$ vs. $25.8(24.5), p<0.001)$, worse functional status on admission (Barthel Index (BI) 13.0 (5.8) vs. 25.6 (24.1), p < 0.001), worse Glasgow Coma Scale $(9.7$ (3.8) vs. 12.0 (3.3), p < 0.001), worse Coma Remission Scale (9.5 (6.4) vs. 14.0 (6.8), p = 0.001), more co-diagnoses (18.8 (5.1) vs. 13.3 (5.5), $p<0.001)$, and higher Patient Clinical Complexity Levels (PCCL). The outcome was significantly worse among ESBL positive patients (BI 28.2 (21.7) vs. 47.4 (31.0), $p$ < 0.001; Early Rehabilitation Index -43.0 (51.7) vs. -26.0 (35.4), p < 0.001). ESBL patients had the same amount of therapy per day (136.2 (20.2) vs. $140.2(18.7) \mathrm{min} /$ day, n.s.), but the overall sum was significantly larger in the ESBL group due to longer LOS $(p<0.001)$. Mortality of both groups was comparable (3.8\% vs. $4.1 \%) .54 .3 \%$ of ESBL negative patients were discharged to home, but only $34.5 \%$ of ESBL colonized. $48 \%$ of ESBL positive patients were discharged to a nursing home, but only $25.1 \%$ of the ESBL free patients. Functional recovery of ESBL carriers undergoing neurological early rehabilitation is worse than that of patients without multidrug-resistant germs. Poorer outcome is not resulting from less therapy due to CP, but from functional status and higher morbidity on admission.
\end{abstract}


Keywords

ESBL, Early Rehabilitation, Outcome, Neurology

\section{Introduction}

It has been shown in a previous study that the functional outcome of early neurological rehabilitation patients colonized with methicillin-resistant Staphylococcus aureus (MRSA) is worse [1]. This finding could not be explained by less therapy due to contact precautions (CP), but by worse functional status and higher morbidity on admission [1].

Apart from MRSA, multidrug-resistant gram negative bacteria producing extended-spectrum beta-lactamases (ESBL) are an increasing problem in neurological rehabilitation [2]. While MRSA prevalence on admission was $11.4 \%$, multidrug-resistant gram negative germs (ESBL) were observed in $11.8 \%$ of early rehabilitation cases [2]. A recent prospective study found an ESBL Enterobacteriaceae (ESBL-PE) prevalence of $26.0 \%$ [3]. Appallingly, $3.5 \%$ of healthcare personnel and $9 \%$ of family members of rehabilitation patients who were carriers of ESBL-PE were also found to be colonized [4]. Risk factors of ESBL-PE colonization are recent stay in acutecare hospital for over two weeks, history of ESBL colonization, unconsciousness on admission, surgery during the last year, and antibiotic treatment during the last month [3]. Other multidrug-resistant gram negative bacteria like Proteus mirabilis (ESBL-PM) also cause problems in rehabilitation [5]. Outbreaks and nosocomial transmissions of ESBL bacteria in neurological rehabilitation have been reported, too [6] [7]. Highly resistant gram negative germs are dreaded on spinal cord rehabilitation wards, because they may cause severe urinary tract infections [7].

In Germany, it has been suggested to dismiss the ESBL nomenclature and to divide multidrug-resistant gram negative (MRGN) germs in 3MRGN or 4MRGN according to their antibiotic resistance (resistant to 3 resp. 4 out of 4 groups of antibitotics: ureidopenicillins, third/fourth generation cephalosporins, carbapenems, quinolones) [8]. Contact precautions (CPs) are generally recommended for hospital inpatients colonized with 4MRGN, while CPs for patients with 3MRGN are only suggested on intensive care units (ICU) and wards with immunosuppressed patients [8].

CPs raise ethical concerns [9] because they may cause psychological distress like depression and anxiety [10]. Apart from depression, CPs account for less patient-healthcare worker contacts (disregard), delays, more noninfectious adverse events and decreased patient satisfaction with care [11].

It is hypothesized that ESBL colonization limits rehabilitation because of $\mathrm{CP}$, but there are no studies focusing on the outcome of ESBL carriers in neurological early rehabilitation yet.

\section{Methods}

The BDH Clinic Hessisch Oldendorf is a neurological acute-care hospital (including stroke unit and intensive care units) and rehabilitation facility in Northern Germany with more than 100 neurological early rehabilitation beds [12]. All patients who were known to be ESBL positive in referring hospitals, have been screened on admission (mainly rectal swabs). In a few patients, ESBL colonization was detected later during their rehabilitation. All patients colonized with $4 \mathrm{MRGN}$ have been isolated while $3 \mathrm{MRGN}$ carriers were only on $\mathrm{CP}$ when they were admitted to an ICU or intermediate care unit (IMC) of the BDH-Clinic Hessisch Oldendorf [8].

To find out whether ESBL colonization had any impact on outcome parameters, medical records of $n=643$ neurological early rehabilitation patients admitted to the BDH Clinic Hessisch Oldendorf in 2010 have been reviewed. Barthel index (BI) [13], Early Rehabilitation Index (ERI) [14], Glasgow Coma Scale (GCS) [15], Coma Remission Scale (CRS) [16], and Early Functional Abilities (EFA) [17] on admission have been included in the analysis. As major outcome parameters, BI and ERBI have been recorded. In addition, length of stay (LOS), morbidity (number of co-diagnoses and Patient Clinical Complexity Level—PCCL [12]), and duration of therapy (physiotherapy, ergotherapy, speech therapy, cognitive therapy) have been analyzed.

Statistical analyses included t-tests for independent samples, univariate analyses of variance, and bivariate Pearson correlations. Differences were regarded as significant with $\mathrm{p}<0.05$.

Local ethics committee (BDH-Clinic Hessisch Oldendorf) had no objections because the study was a retros- 
pective database analysis, only (no intervention).

\section{Results}

Among 643 neurological early rehabilitation patients $(\mathrm{n}=298$ (female); $\mathrm{n}=345$ (male)), $\mathrm{n}=148(23.0 \%)$ were ESBL positive on admission and/or during early rehabilitation. ESBL positive patients were on CP for a mean of 19.2 (27.2) days, range 0 to 138 days. Most patients admitted to neurological early rehabilitation suffered from an ischemic stroke (Table 1). Most of the gram negative ESBL producing bacteria were enterobacteriaceae (60.9\%: Klebsiella pneumoniae $>$ Escherichia coli $>$ Serratia marcescens/Proteus mirabilis), 39.1\% Pseudomonas aeruginosa.

ESBL positive were not older than negative patients (Table 2). Among ESBL carriers, age correlated nega-

Table 1. Main diagnoses of ESBL positive and negative neurological early rehabilitation patients.

\begin{tabular}{ccc}
\hline & ESBL positive & ESBL negative \\
\hline Ischemic stroke & $45(30.4 \%)$ & $201(40.6 \%)$ \\
Intracranial hemorrhage & $41(27.7 \%)$ & $86(17.4 \%)$ \\
Hypoxia & $7(4.7 \%)$ & $13(2.6 \%)$ \\
Brain tumour & $6(4.1 \%)$ & $14(2.8 \%)$ \\
Polyneuropathy/Guillian-Barre-Syndrome & $5(3.4 \%)$ & $14(2.8 \%)$ \\
Spinal injury & $5(3.4 \%)$ & $6(1.2 \%)$ \\
Brain injury & $2(1.4 \%)$ & $14(6.8 \%)$ \\
Other main diagnosis & $37(25.0 \%)$ & $147(29.7 \%)$ \\
Sum & $148(100 \%)$ & $495(100 \%)$ \\
\hline
\end{tabular}

Table 2. Characteristics of ESBL positive and negative neurological early rehabilitation patients.

\begin{tabular}{|c|c|c|c|}
\hline & ESBL positive & ESBL negative & p-value* \\
\hline Age [years] & $64.5(15.4)$ & $67.0(15.8)$ & n.s. \\
\hline LOS-neurological early rehabilitation [days] & $67.5(42.0)$ & $25.8(24.5)$ & $<0.001$ \\
\hline LOS-entire neurological rehabilitation [days] & $84.6(47.4)$ & $46.8(47.1)$ & $<0.001$ \\
\hline Number of co-diagnoses [n] & $18.8(5.1)$ & $13.3(5.5)$ & $<0.001$ \\
\hline Barthel Index (BI) on admission [0 to 100] & $13.0(5.8)$ & $25.6(24.1)$ & $<0.001$ \\
\hline Barthel index on discharge [ 0 to 100$]$ & $28.2(21.7)$ & $47.4(31.0)$ & $<0.001$ \\
\hline Early Rehabilitation Index (ERI) on admission $[-325$ to 0$]$ & $-72.4(57.8)$ & $-47.9(47.6)$ & $<0.001$ \\
\hline ERI on discharge $[-325$ to 0$]$ & $-43.0(51.7)$ & $-26.0(35.4)$ & $<0.001$ \\
\hline Coma Remission Scale (CRS) [0 to 24] on admission & $9.5(6.4)$ & $14.0(6.8)$ & $<0.01$ \\
\hline Glasgow Coma Scale (GCS) [3 to 15] on admission & $9.7(3.8)$ & $12.0(3.3)$ & $<0.001$ \\
\hline Early functional abilities (EFA)-vegetative [4 to 20] & $9.2(3.4)$ & $12.4(7.0)$ & $<0.001$ \\
\hline EFA-faciooral [4 to 20] & $11.1(6.0)$ & $15.4(5.3)$ & $<0.001$ \\
\hline EFA-sensorimotor [7 to 35$]$ & $16.0(7.4)$ & $22.8(8.2)$ & $<0.001$ \\
\hline EFA-cognitive [5 to 25] & $14.7(6.7)$ & $18.7(5.2)$ & $<0.001$ \\
\hline Physiotherapy [min/day] & $41.5(12.2)$ & $36.6(10.2)$ & $<0.001$ \\
\hline Ergotherapy [min/day] & $30.6(2.2)$ & $31.2(4.7)$ & n.s. \\
\hline Speech therapy [min/day] & $24.5(5.8)$ & $27.2(5.7)$ & $<0.001$ \\
\hline Cognitive therapy [min/day] & $39.1(9.1)$ & $42.9(9.4)$ & $<0.001$ \\
\hline Total main therapies [min/day] & $136.2(20.2)$ & $140.2(18.7)$ & n.s. \\
\hline
\end{tabular}

\footnotetext{
${ }^{*}$ t-tests for independent samples; n.s. $=$ not significant $(\mathrm{p}>0.05)$.
} 
tively with changes in BI $(r=-0.183, p<0.05$, Figure 1$)$ and ERI $(r=-0.173, p<0.01$, Figure 2$)$. LOS in neurological rehabilitation was considerably longer among ESBL positives (Table 2). BI, ERI, GCS and EFA on admission were worse in the ESBL group. In addition, numbers of co-diagnoses and PCCL among these patients were significantly higher (Table 2 and Table 3). Improvements in BI were significantly smaller in the ESBL positive group: $+15.2(21.1)$ vs. $+21.8(25.7), \mathrm{p}<0.01$. ESBL carriers had suffered a polytrauma more frequently than ESBL negative patients (19.0\% vs. $10.9 \%$, Table 4$)$.

BL positive neurological early rehabilitation patients had significantly more physiotherapy but less speech and cognitive therapy per day (Table 2). Ergotherapy did not differ between the groups. However, duration of all therapies did not differ (136.2 (20.2) vs. 140.2 (18.7) $\mathrm{min} / \mathrm{d}, \mathrm{p}>0.05)$. Because of significantly longer LOS

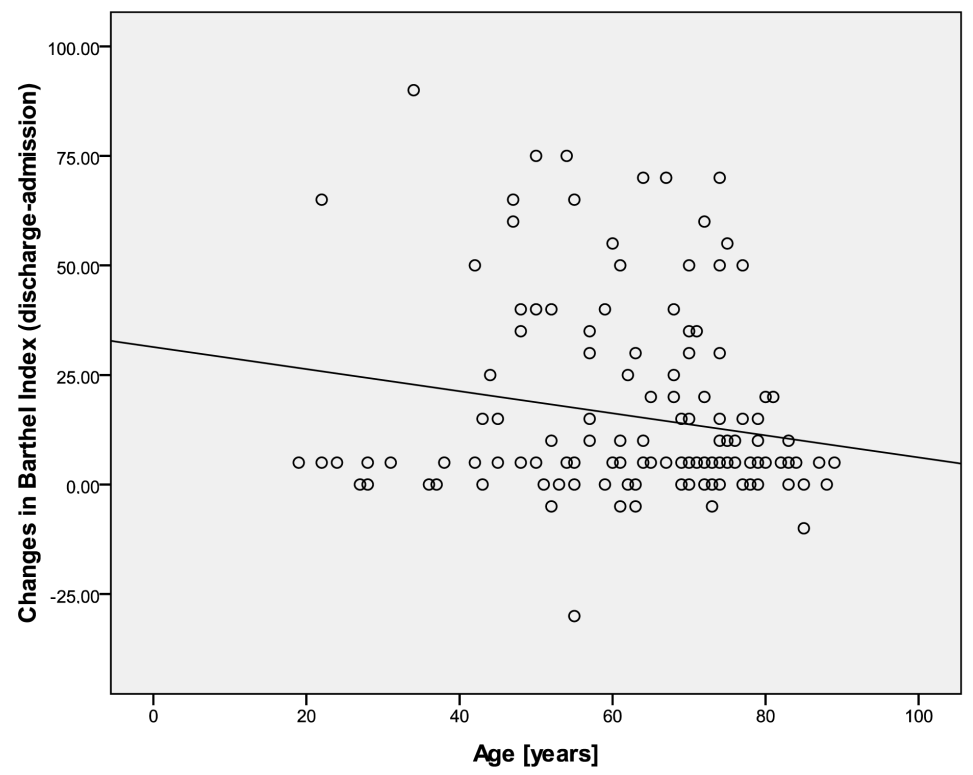

Figure 1. Age correlated negatively with changes in Barthel Index (discharge minus admission) among ESBL colonized patients.

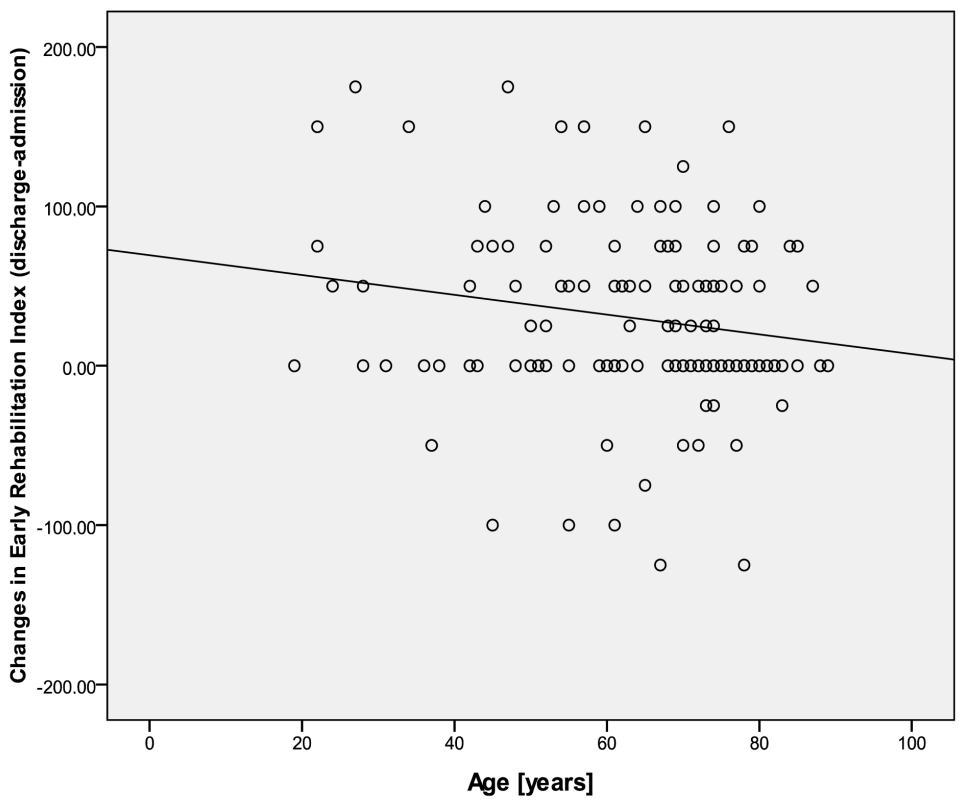

Figure 2. Age correlated negatively with changes in Early Rehabilitation Index (discharge minus admission) among ESBL colonized patients. 
Table 3. PCCL (Patient Clinical Complexity Level) of ESBL positive and negative patients.

\begin{tabular}{ccccc}
\hline & & ESBL & & Sum \\
\cline { 3 - 4 } & & Negative & positive & 35 \\
PCCL & 0 & 35 & 0 & 15 \\
& 1 & 15 & 0 & 48 \\
& 2 & 43 & 5 & 307 \\
& 3 & 255 & 52 & 238 \\
\hline
\end{tabular}

$\chi^{2}=56.8(\mathrm{p}<0.001)$.

Table 4. Medical history of polytrauma among ESBL positive and negative neurological early rehabilitation patients.

\begin{tabular}{|c|c|c|c|c|}
\hline & & \multicolumn{2}{|c|}{ Polytrauma } & \multirow{2}{*}{ Sum } \\
\hline & & no & yes & \\
\hline \multirow{2}{*}{ ESBL } & negative & 441 & 54 & 495 \\
\hline & positive & 119 & 28 & 147 \\
\hline \multicolumn{2}{|c|}{ Sum } & 560 & 82 & 642 \\
\hline
\end{tabular}

$\chi^{2}=6.7(\mathrm{p}<0.01)$

of ESBL positive patients, the overall sum of therapeutic procedures during early rehabilitation was considerably higher in this group.

While mortality was comparable (3.8\% vs. $4.1 \%), 54.3 \%$ of ESBL negative patients were discharged to home, but only $34.5 \%$ of ESBL colonized (Table 5). Nearly half of the ESBL positives (48\%) were discharged to a nursing home, but only a fourth of the ESBL free patients $(25.1 \%)$.

A univariate analysis of variance (ANOVA) was performed using the following model: changes in BI (discharge minus admission) as dependent variable; colonization with ESBL and PCCL as categorical independent variables; age, BI on admission, GCS on admission, CRS on admission, duration of isolation, physiotherapy, ergotherapy, speech therapy and cognitive therapy per day as independent covariates. This model explained $64.0 \%$ of the data variation $(\mathrm{p}<0.001)$. PCCL had a highly significant influence $(\mathrm{p}<0.001)$, but not ESBL colonization itself. Age $(p<0.01)$, BI on admission $(p<0.001)$ and physiotherapy per day $(p<0.01)$ also had a significant influence on changes in BI. CRS, GCS, ergotherapy, speech and cognitive therapy did not have an impact on BI changes.

\section{Discussion}

Mutidrug-resistant bacteria like MRSA or ESBL producing gram negative germs are a growing problem in neurological rehabilitation because many of these patients are on $\mathrm{CP}$, in particular on ICU. Up to $26.0 \%$ of patients admitted to rehabilitation may be colonized by ESBL producing bacteria [3]. In neurological early rehabilitation colonization rates of $11.2 \%$ in a retrospective study have been published [2]. It has been shown that neurological early rehabilitation patients colonized by MRSA have a significantly worse outcome [1]. This finding could not be explained by less therapy due to CP but by worse functional status on admission and higher morbidity [1]. However, no data on the outcome of ESBL colonized neurological early rehabilitation patients is available, yet.

In the present study, there was a rate of 23.0\% (148/643) ESBL carriers among neurological early rehabilitation patients what comes close to the rate of previously published $26.0 \%$ [3]. However, it has to be pointed out 
Table 5. Discharge placement.

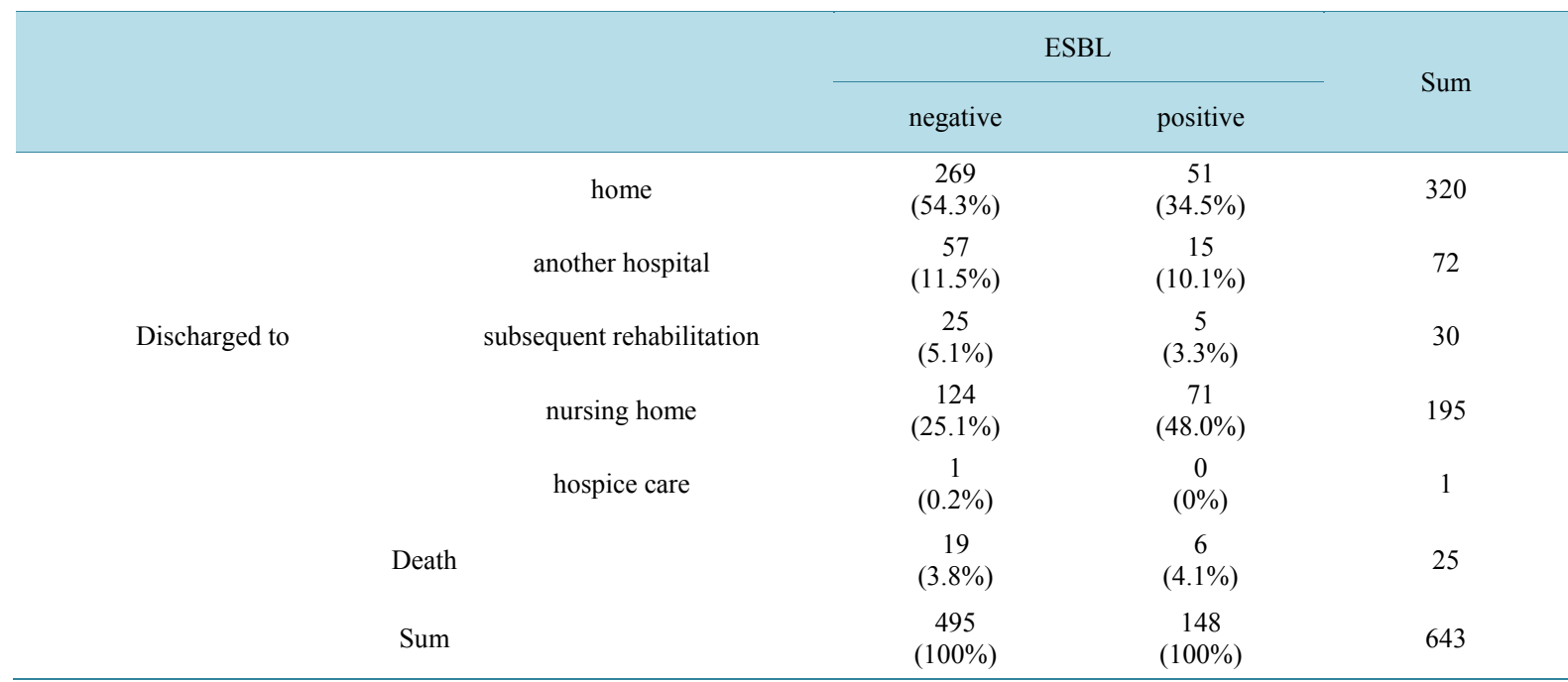

$\chi^{2}=30.1(\mathrm{p}<0.001)$

that the present study had a retrospective design and not all patients admitted to rehabilitation have been screened for gram negative multidrug-resistant bacteria. Thus, the real colonization rate might even be higher.

Patients were on CP for a mean of 19.2 (27.2) days. This finding is surprising because duration of isolation due to MRSA is considerably longer (about 31.5 days [3]) and ESBL producing bacteria may not be decolonized successfully [18]. The huge standard deviation of CP duration indicates that not all of the ESBL colonized patients were on CP. In Germany, only a fraction of patients with gram negative multidrug-resistant germs needs to be isolated, in particular on ICU or wards with immunosuppressed patients [8].

It is well known that age has an impact on the outcome of neurological patients, e.g. after stroke [1] [19]. The present study also demonstrated a negative correlation between age and improvement in activities of daily living (BI) suggesting that age is a risk factor for poor neurological outcome. However, ESBL positive and negative groups did not differ with respect to age.

In line with previous studies, ESBL positive patients had a higher morbidity (PCCL, number of co-diagnoses) and functional status (Barthel index, ERBI, CRS, EFA, GCS) was worse on admission. Like with MRSA [1], LOS was significantly longer among ESBL carriers which may be explained by considerably higher morbidity and worse functional status.

Surprisingly, overall sum of therapy was significantly larger in the ESBL group which can be explained by longer LOS in this cohort. Intensity of therapy (min per day), however, did not differ between ESBL negative and positive patients. This finding suggests that ESBL colonized patients on CP do not necessarily receive less therapy than ESBL negative patients. Even so, the outcome of ESBL carriers was worse. How can this finding be explained? It is well known that low functional status on admission and co-morbidity are risk factors for poor outcome [14] [20]. It emerges from literature that BI in the early phase is a strong predictor for long-term functional outcome [21]. Poor outcome among ESBL carriers may be explained by worse functional status and higher morbidity on admission. In addition, lower BI and ERBI values on admission account for longer LOS among ESBL carriers [14] [20]. This hypothesis is confirmed by a univariate analysis of variance: It turned out that BI improvement among ESBL positives was strongly influenced by PCCL (as a measure of morbidity), age, and BI on admission. ESBL colonization itself had no independent influence on BI changes. Further studies on this topic are strongly encouraged.

\section{Conclusion}

The outcome of ESBL colonized early neurological rehabilitation patients is worse than functional independence of ESBL free patients. This finding can't be explained by less therapy due to CP, but by significantly worse functional status and morbidity of ESBL carriers on admission. 


\section{Competing Interests}

The author declares that he has no financial or non-financial competing interests.

\section{Authors' Contributions}

JDR, as the only author designed the database, made all statistical analyses, and drafted the paper.

\section{Acknowledgements}

The author thanks Ms. Pauline Scholz, study nurse, for her work on the database and reviewing the medical records.

\section{References}

[1] Rollnik, J.D. (2014) Outcome of MRSA Carriers in Neurological Early Rehabilitation. BMC Neurology, 14, 34. http://dx.doi.org/10.1186/1471-2377-14-34

[2] Rollnik, J.D. (2014) Mulitdrug-Resistant Germs in Neurological Early Rehabilitation (2004-2013). Rehabilitation (Stuttg), in Press.

[3] Bilavsky, E., Temkin, E., Lerman, Y., Rabinovich, A., Salomon, J., Lawrence, C., Rossini, A., Salvia, A., Samso, J.V., Fierro, J., Paul, M., Hart, J., Gniadkowski, M., Hochman, M., Kazma, M., Klein, A., Adler, A., Schwaber, M.J. and Carmeli, Y. (2014) The MOSAR WP5 Study Team: Risk Factors for Colonization with Extended-Spectrum BetaLactamase-Producing Enterobacteriaceae on Admission to Rehabilitation Centres. Clinical Microbiology and Infection, 20, O804-O810. http://dx.doi.org/10.1111/1469-0691.12633

[4] Adler, A., Baraniak, A., Izdebski, R., Fiett, J., Salvia, A., Samso, J.V., Lawrence, C., Solomon, J., Paul, M., Lerman, Y., Schwartzberg, Y., Mordechai, E., Rossini, A., Fierro, J., Lammens, C., Malhotra-Kumar, S., Goossens, H., Hryniewicz, W., Brun-Buisson, C., Gniadkowski, M. and Carmeli, Y. (2014) MOSAR Team: A Multinational Study of Colonization with Extended Spectrum $\beta$-Lactamase-Producing Enterobacteriaceae in Healthcare Personnel and Family Members of Carrier Patients Hospitalized in Rehabilitation Centres. Clinical Microbiology and Infection, 20, O516O523. http://dx.doi.org/10.1111/1469-0691.12560

[5] Adler, A., Baraniak, A., Izdebski, R., Fiett, J., Gniadkowski, M., Hryniewicz, W., Salvia, A., Rossini, A., Goossens, H., Malhotra, S., Lerman, Y., Elenbogen, M. and Carmeli, Y. (2013) MOSAR WP5 \& WP2 Study Groups: A Binational Cohort Study of Intestinal Colonization with Extended-Spectrum $\beta$-Lactamase-Producing Proteus mirabilis in Patients Admitted to Rehabilitation Centres. Clinical Microbiology and Infection, 19, E51-E8. http://dx.doi.org/10.1111/1469-0691.12072

[6] Holländer, R., Ebke, M., Barck, H. and von Pritzbuer, E. (2001) Asymptomatic Carriage of Klebsiella pneumoniae Producing Extended-Spectrum Beta-Lactamase by Patients in a Neurological Early Rehabilitation Unit: Management of an Outbreak. Journal of Hospital Infection, 48, 207-213. http://dx.doi.org/10.1053/jhin.2001.0997

[7] Slim, E., Smit, C.A., Bos, A.J. and Peerbooms, P.G. (2009) Nosocomial Transmission of Highly Resistant Microorganisms on a Spinal Cord Rehabilitation Ward. Journal of Spinal Cord Medicine, 32, 422-427.

[8] Kommission für Krankenhaushygiene und Infektionsprävention (KRINKO) beim Robert-Koch-Institut (RKI) (2012) Hygienemaßnahmen bei Infektionen oder Besiedelung mit multiresistenten gramnegativen Stäbchen. Bundesgesundheitsbl-Gesundheitsforsch-Gesundheitsschutz, 55, 1311-1354. http://dx.doi.org/10.1007/s00103-012-1549-5

[9] Pike, J.H. and McLean, D. (2002) Ethical Concerns in Isolating Patients with Methicillin-Resistant Staphylococcus Aureus on the Rehabilitation Ward: A Case Report. Archives of Physical Medicine and Rehabilitation, 83, 1028-1030. http://dx.doi.org/10.1053/apmr.2002.33108

[10] Tarzi, S., Kennedy, P., Stone, S. and Evans, M. (2001) Methicillin-Resistant Staphylococcus Aureus: Psychological Impact of Hospitalization and Isolation in an Older Adult Population. Journal of Hospital Infection, 49, $250-254$. http://dx.doi.org/10.1053/jhin.2001.1098

[11] Morgan, D.J., Diekema, D.J., Sepkowitz, K. and Perencevich, E.N. (2009) Adverse Outcomes Associated with Contact Precautions: A Review of the Literature. American Journal of Infection Control, 37, 85-93. http://dx.doi.org/10.1016/j.ajic.2008.04.257

[12] Rollnik, J.D. and Janosch, U. (2010) Current Trends in the Length of Stay in Neurological Early Rehabilitation. Deutsches Ärzteblatt International, 107, 286-292.

[13] Mahoney, F.I. and Barthel, D.W. (1965) Functional Evaluation: The Barthel Index. Maryland State Medical Journal, 14, 61-65.

[14] Rollnik, J.D. (2011) The Early Rehabilitation Barthel Index (ERBI). Rehabilitation, 50, 408-411. 
http://dx.doi.org/10.1055/s-0031-1273728

[15] Teasdale, G. and Jennett, B. (1974) Assessment of Coma and Impaired Consciousness. A Practical Scale. The Lancet, 2 , 81-83. http://dx.doi.org/10.1016/S0140-6736(74)91639-0

[16] Ortega-Suhrkamp, E. and von Wild, K.R. (2002) Standards of Neurologic-Neurosurgical Early Rehabilitation-A Concept of the Study Group Neurological-Neurosurgical Early Rehabilitation. Acta Neurochirurgica Supplement, 79, 11-19.

[17] Alvsåker, K., Walther, S.M., Kleffelgård, I., Mongs, M., Drægebø, R.A. and Keller, A. (2011) Inter-Rater Reliability of the Early Functional Abilities Scale. Journal of Rehabilitation Medicine, 43, 892-899.

[18] Huttner, B., Haustein, T., Uçkay, I., Renzi, G., Stewardson, A., Schaerrer, D., Agostinho, A., Andremont, A., Schrenzel, J., Pittet, D. and Harbarth, S. (2013) Decolonization of Intestinal Carriage of Extended-Spectrum $\beta$-LactamaseProducing Enterobacteriaceae with Oral Colistin and Neomycin: A Randomized, Double-Blind, Placebo-Controlled Trial. Journal of Antimicrobial Chemotherapy, 68, 2375-2382.

[19] Kwakkel, G. and Kollen, B.J. (2013) Predicting Activities after Stroke: What Is Clinically Relevant? International Journal of Stroke, 8, 25-32. http://dx.doi.org/10.1111/j.1747-4949.2012.00967.x

[20] Pettersen, R., Dahl, T. and Wyller, T.B. (2002) Prediction of Long-Term Functional Outcome after Stroke Rehabilitation. Clinical Rehabilitation, 16, 149-159. http://dx.doi.org/10.1191/0269215502cr482oa

[21] Rollnik, J.D. (2009) Barthel-Index as a Length of Stay Predictor in Neurological Rehabilitation. Rehabilitation, 48, 9194. http://dx.doi.org/10.1055/s-0029-1202294

\section{List of Abbreviations}

\begin{tabular}{ll}
\hline BI & Barthel Index \\
CP & Contact Precautions (Isolation) \\
CRS & Coma Remission Scale \\
EFA & Early Functional Abilities \\
ERI & Early Rehabilitation Index \\
ESBL & Extended-Spectrum Beta-Lactamase \\
GCS & Glasgow Coma Scale \\
ICU & Intevsive Care Unit \\
IMC & Intermediate Care \\
LOS & Length of Stay \\
MRGN & Multidrug-Resistatant Gram Negative \\
MRSA & Methicillin-Resistant Staphylococcus aureus \\
PCCL & Patient Clinical Complexity Level \\
\hline
\end{tabular}

